\title{
Magnetic Stimulation of Mammalian Peripheral Nerves In Vivo: An Alternative to Functional Electrical Stimulation
}

\author{
Zachary B. Kagan ${ }^{1}$, Anil Kumar RamRakhyani ${ }^{2}$, Faisal Khan ${ }^{2}$, Gianluca Lazzi², \\ Richard A. Normann ${ }^{1}$, and David J. Warren ${ }^{1}$
}

\begin{abstract}
Functional electrical stimulation is the current gold standard for stimulating neuronal interfaces for functional neuromuscular and cortical applications, but it is not without its drawbacks. One such fault is the need to have direct electrical contact with the nerve tissue, and any side effects this causes. Functional magnetic stimulation, which works though electromagnetic induction, does not require electrical contact and may be a viable alternative to functional electrical stimulation. We are investigating the capabilities of magnetic stimulation with centimeter scale $(<2.5 \mathrm{~cm})$ coils in feline and rodent sciatic nerves in vivo. We have shown that magnetic stimulation can consistently produce the same levels of neuromuscular activation as electrical stimulation. Additionally, the position of the coil relative to the nerve influences neuromuscular activation, suggesting the possibility of selective muscle activation.
\end{abstract}

\section{INTRODUCTION}

In recent decades, several modalities of neuromuscular tissue stimulation have been investigated, including functional electrical stimulation (FES) via cuff electrodes [1] and penetrating electrodes [2], functional magnetic stimulation (FMS) [3], and others like sieve electrodes and infrared optical stimulation $[4,5]$. While each modality has applications it is best suited for, electrical neurostimulating devices are most commonly chosen, likely due to their ease of evoking responses [4]. Despite their successes, there are still challenges when using electrically stimulating implanted devices in long term studies, potentially due to the foreign body response from neural tissue [6]. This foreign body response is proposed to be the result of accumulating proteins and macrophages at the implant site, which separates the electrode from neural tissue by surrounding the electrode with scar tissue [7]. This walling off of the electrode reduces the effectiveness of stimulation and recording with that electrode, requiring larger and potentially unsafe levels of stimulation to evoke a response [8]. Furthermore, the degradation of the electrode reduces the stimulation effectiveness and exacerbates the foreign body response. To address this, a stimulation modality that does not require direct electrical contact to tissue, functional magnetic stimulation, is being investigated. Hence, the foreign body response associated with current injection via an electrode may be reduced when

This material is based upon work supported by the National Science Foundation under grant No. ECCS-1202235.

${ }^{1}$ Zachary B. Kagan, Richard A. Normann, and David J. Warren are with the Department of Bioengineering, University of Utah, Salt Lake City, UT 84112, USA

${ }^{2}$ Anil Kumar RamRakhyani, Faisal Khan, and Gianluca Lazzi are with the Department of Electrical and Computer Engineering, University of Utah, Salt Lake City, UT 84112, USA using FMS, allowing an implanted coil to remain effective for longer periods than electrodes.

Magnetic stimulation of peripheral neural tissue uses time-varying electromagnetic fields to activate motor and sensory nerve fibers, which then produce muscle activation and percepts, respectively. However, magnetic stimulation is arguably better known as a research and treatment tool, particularly in corticospinal regions in the form of transcranial magnetic stimulation (TMS) [9]. The solenoid coils used for TMS often have outer diameters larger than $50 \mathrm{~mm}$ [10], but a coil that size is impractical as an implanted device. We are investigating smaller coils starting with outer diameters under $25 \mathrm{~mm}$ and intend to progress to yet smaller microcoils. Such smaller coils have been shown to be effective at exciting smaller volumes of tissue [11], and thus may provide the levels of selectivity observed with penetrating electrodes.

\section{Methods}

\section{A. Coil Fabrication and Stimulation Circuit}

A hand wound solenoid coil was produced with 19 AWG magnet wire to have 6 turns of wire per layer and 5 layers of wire for a total of 30 turns. The coil's outer diameter was $23.5 \mathrm{~mm}$, and inner diameter was $9.5 \mathrm{~mm}$. The total height of the coil was $8.75 \mathrm{~mm}$. Large, fast-transient currents, on the order of $1 \mathrm{kA}$, were necessary to evoke a measurable neuromuscular response, as the time varying magnetic field, which created the electric field in the tissue, was produced by the time varying current [3]. To generate these currents, a $2.2 \mathrm{mF}$ capacitor was charged via a high-voltage DC power supply and discharged through the coil with custom power electronics similar to previous designs for other magnetic stimulation applications [3, 12]. The time course of the current through the coil after discharge was an underdamped sinusoid, with an effective pulse duration of approximately $180 \mu \mathrm{s}$. The pulse duration was defined as the time from the start of stimulation to the first instance the time derivative of the current crossed zero.

\section{B. Surgical and Experimental Procedures for Testing Mag- netic Stimulation Efficacy}

All procedures were approved by the University of Utah Institutional Animal Care and Use Committee. SpragueDawley rats $(250-400 \mathrm{~g})$ were used in rodent sciatic nerve preparations $(\mathrm{n}=2)$, and anesthesia was induced and maintained with Isofluorane gas (1-5\%). In feline sciatic nerve preparations $(n=2)$, anesthesia was induced with Telazol $(9$ 
to $12 \mathrm{mg} / \mathrm{kg} \mathrm{IM}$ ) and maintained with Isofluorane gas (1$2 \%$ ). For both feline and rodent preparations, vital signs (heart rate, blood oxygen saturation, and rectal temperature) were monitored to assess the depth of anesthesia and animal condition. Surgical procedures for exposing the sciatic nerve followed those previously described [2]. The stimulating coil, which was insulated from tissue by a $2 \mathrm{~mm}$ thick glass plate, was positioned and fixed in place directly above the nerve approximately halfway between the hip and knee with a stereotactic 3-dimension positioning system. The stimulating system's capacitor discharge voltages ranged from 80 to 350 $\mathrm{V}$ in $20 \mathrm{~V}$ increments in felines and $10 \mathrm{~V}$ increments in rodents. In felines, the coil was moved in 2-mm increments over a 34-mm range in a direction transverse to the length of the nerve using the positioning system. Coil origin $(0$ $\mathrm{mm}$ ) is defined as the coil position where the anterior edge of the nerve is tangent to the posterior edge of the coil. Positive coil positions indicate the coil has moved anterior, while negative coil positions indicate the coil has moved posterior, and is partially covering the nerve or is entirely posterior to the nerve from this orientation. A single trial was recorded at each voltage-position pair in felines. Six trials were recorded at every voltage in rodents. To normalize the magnetic stimulation response, electrical stimulation trials were performed by placing an isolated bipolar hook electrode on the nerve and stimulating with monophaisc (cathodic distal) voltage pulses for $200 \mu$ s at a voltage between 0.1 and $1 \mathrm{~V}$ in $0.1 \mathrm{~V}$ increments.

Electromyography (EMG) data was recorded differentially from the major extensor and flexor muscles of the ankle, lateral gastrocnemius (LG), medial gastrocnemius (MG), tibialis anterior (TA), and soleus (Sol). EMG wires were made as described in prior work [13] and inserted as pairs into each muscle. EMG recordings were bandpass filtered between $10 \mathrm{~Hz}$ to $1 \mathrm{kHz}$ with a differential $\mathrm{AC}$ amplifier. Amplifier ground was provided via a $\mathrm{Ag} / \mathrm{AgCl} 15 \mathrm{AWG}$ wire inserted under the skin near the hip. The animal's ipsilateral foot was secured to a six-axis force and torque transducer which was fixed to the surgery table to record forces and torques produced by stimulation (force and torque data not shown here). Data was recorded using a Cerebus data acquisition system at $30 \mathrm{kHz}$.

\section{RESULTS}

\section{A. FMS and FES Produce Similar Neuromuscular Activation}

To compare the nature and similarity of innervated muscular responses evoked by FES and FMS, we recorded the EMG signals in response to stimulating with a coil and a bipolar hook electrode, respectively, over a range of stimulation strengths. As expected, FMS resulted in EMG responses with similar magnitudes, latencies, and kinetics as those observed with FES. For example, we observed near identical EMG responses from TA in a feline after stimulating with both FES and FMS at stimulating intensities slightly above threshold, at intermediate levels, and near maximal levels as shown in Fig. 1. This is consistent with the idea that the nerve fibers are being recruited in a similar manner. To see whether the graded response was maintained as stimulus intensity increased, we examined the range (max. - min.) of each of the 4 EMG signals in a window 2 to $16 \mathrm{~ms}$ after stimulation (Fig. 2). FES (left) and FMS (right) both exhibited sigmoidal recruitment curves, similar maximal activation values, and consistent levels of activation.

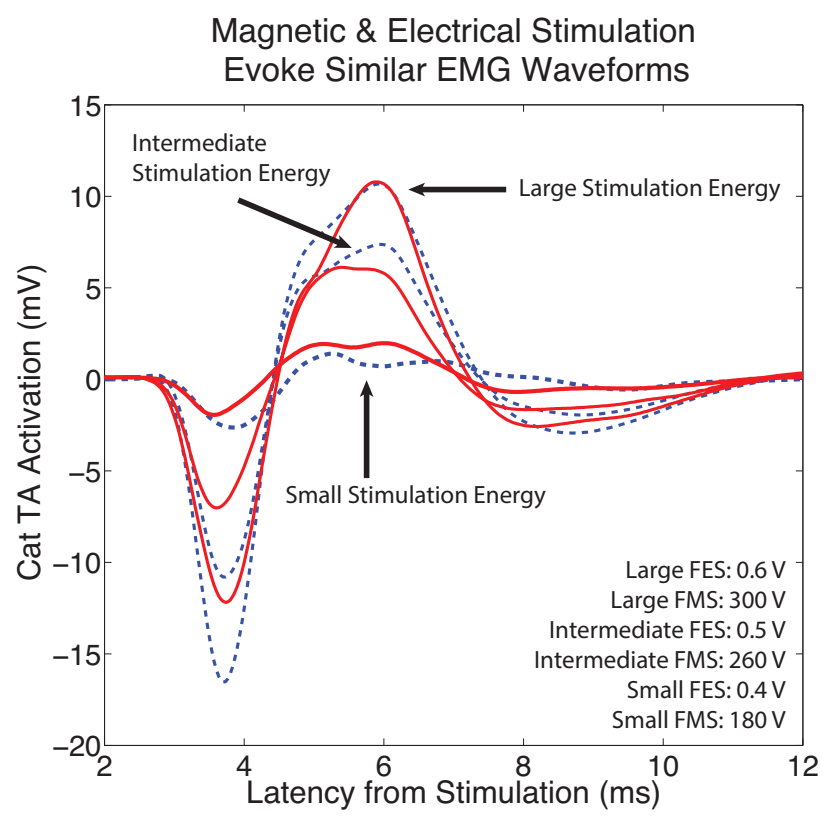

Fig. 1. Feline tibialis anterior (TA) compound muscle action potential from functional electrical stimulation (dashed lines) at stimulation voltages of $0.4 \mathrm{~V}, 0.6 \mathrm{~V}$, and $0.8 \mathrm{~V}$ and functional magnetic stimulation (solid lines) at stimulation voltages of $180 \mathrm{~V}, 260 \mathrm{~V}$, and $300 \mathrm{~V}$. Muscle response transitions from near threshold levels (Small Energy lines) to intermediate levels (Intermediate Energy lines) to near maximal levels (Large Energy lines). Each arrow points to one trace from each modality.

\section{B. FMS Produces Consistent Neuromuscular Activation}

To determine the consistency of responses from FES, six trials were recorded for each stimulation voltage, from 100 to $240 \mathrm{~V}$ in $10 \mathrm{~V}$ increments, in a rodent. A recruitment curve of the rodent's TA response, using the mean response amplitude and standard error of the mean (SEM) is shown in Fig. 3. The standard error of the mean for all levels of stimulation was less than $0.5 \mathrm{mV}$, representing about $1.3 \%$ of the range of responses observed, except for a single stimulation level, $160 \mathrm{~V}$. As the recruitment curve is steepest at this location, it is not surprising that these trials would produce the most variable responses. Even at this stimulation intensity, the SEM was $1.08 \mathrm{mV}$, representing about $2.9 \%$ of the range of the responses observed. These data indicate that FMS can readily produce a graded yet reliable response over the threshold to maximal response range.

\section{FMS can Provide Muscular Selectivity}

Next, we examined the extent to which it is possible to achieve selective muscle activation by moving the coil in a direction perpendicular to the length of the nerve. As the position of the coil was changed, the recruitment curve shifted, indicating that larger voltages were required 


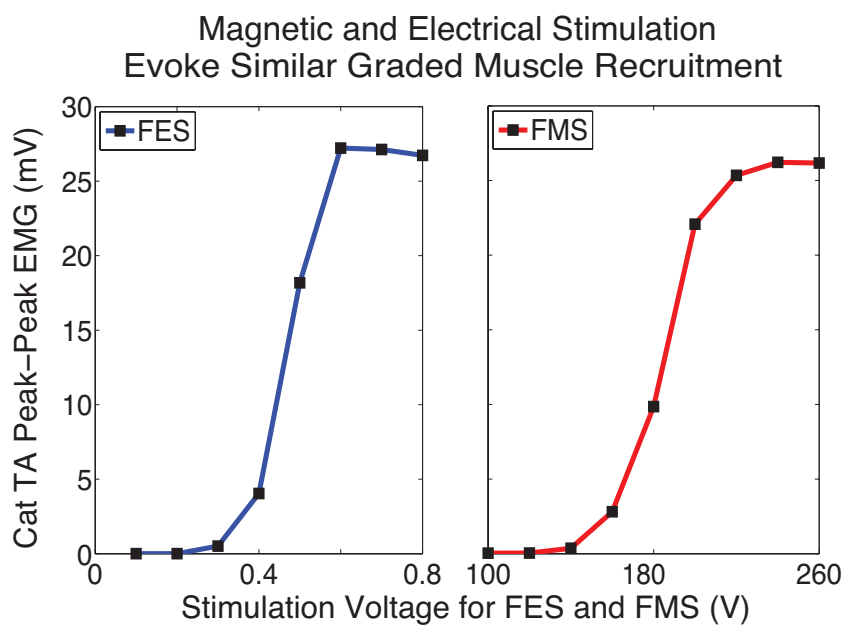

Fig. 2. Feline tibialis anterior compound muscle action potential maxminus-min values from 2 to $16 \mathrm{~ms}$ post-stimulus for functional electrical stimulation (left) and functional magnetic stimulation (right). FES is from 0.1 to $1 \mathrm{~V}$ in $0.1 \mathrm{~V}$ increments. FMS is from 100 to $260 \mathrm{~V}$ in $20 \mathrm{~V}$ increments.

\section{Magnetic Stimulation Evokes Consistent Responses}

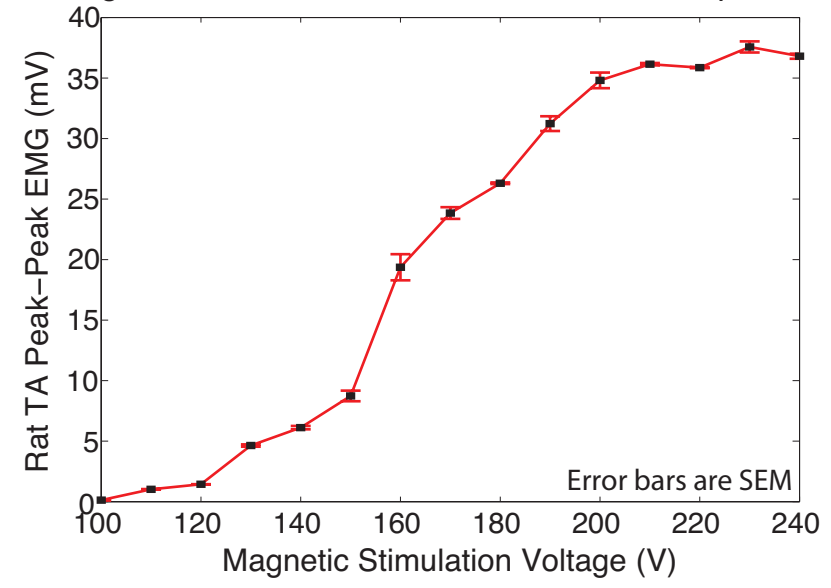

Fig. 3. Rodent tibialis anterior recruitment curve from 100 to $240 \mathrm{~V}$ in $10 \mathrm{~V}$ increments. Six trials were recorded at each voltage increment. Error bars represent standard error of the mean (SEM). All but one response at a given stimulation voltage have SEMs below $0.5 \mathrm{mV}$, indicating excellent consistency. The largest response SEM is $1.08 \mathrm{mV}$, which is still less than $4 \%$ of the range of observed responses.

to activate feline TA as shown in Fig. 4. When the responses from multiple muscles are compared against each other, it becomes clear that FMS can selectively activate muscles. (See Fig. 5). When the coil was positioned at $-16 \mathrm{~mm}$, TA could be selectively activated, indicated by the large difference between TA and LG as the stimulation voltage was increased. Conversely, when the coil was positioned at -12 $\mathrm{mm}, \mathrm{LG}$ can be selectively activated by the same principle.

\section{Discussion}

\section{A. Comparison of FMS and FES}

Despite being different modalities, FES and FMS both produce local neural activation by exploiting the same biophysical phenomenon and developing a driving force along
Coil Position Affects Recruitment Curve Voltages

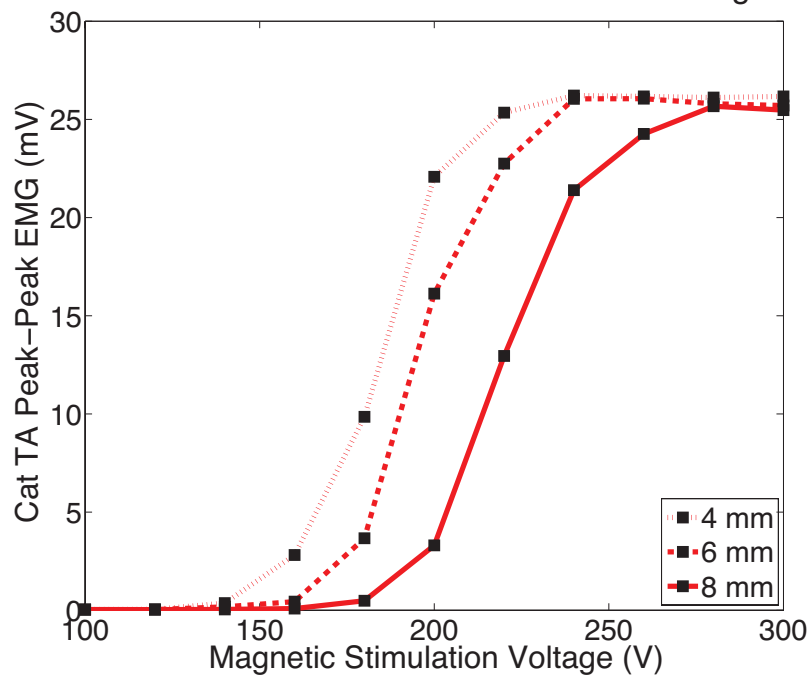

Fig. 4. Recruitment curves for coil positions of $4 \mathrm{~mm}$ (dotted), 6 $\mathrm{mm}$ (dashed), and $8 \mathrm{~mm}$ (solid). Moving the coil anterior increases the stimulation voltages necessary to produce tibialis anterior activation, without changing the general shape of the curve or maximum level of activation.
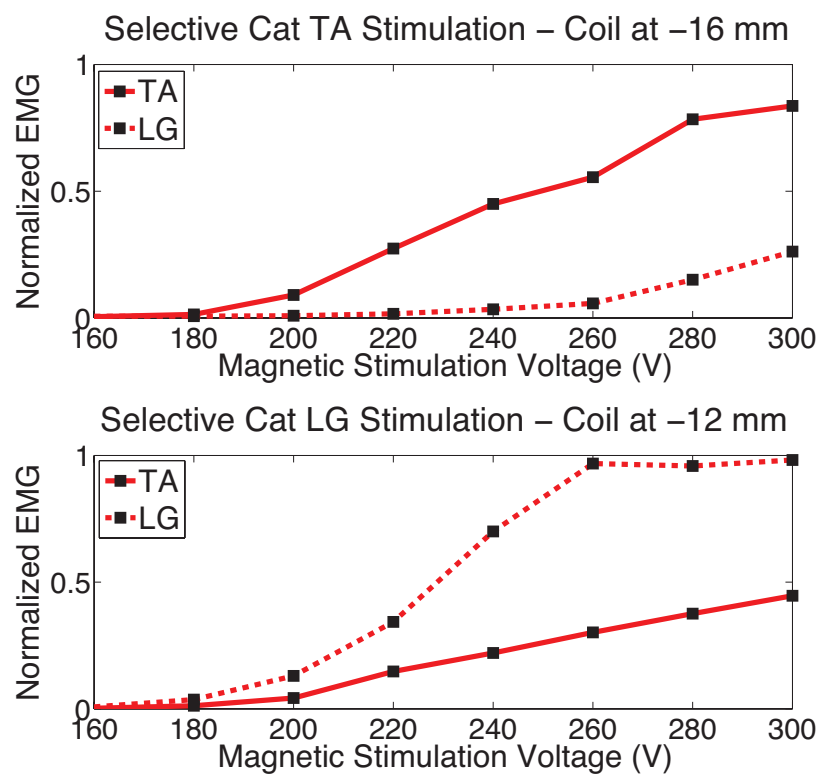

Fig. 5. Feline lateral gastrocnemius (dashed) and tibialis anterior (solid) recruitment curves from $100 \mathrm{~V}$ to $300 \mathrm{~V}$ with coil positions of $-16 \mathrm{~mm}$ (top) and $-12 \mathrm{~mm}$ (bottom). All responses are normalized to that muscle's maximal response across all trials.

the length of the nerve [14]. As a result, it is not surprising the raw EMG responses they produce appear similar (Fig. 1). Furthermore, given that they are activated in similar ways, it is reassuring to observe sigmoidal recruitment curves with similar maximal levels (Fig. 2). In these cases, FES required about twice the threshold stimulation voltage to reach its maximal response ( $0.3 \mathrm{~V}$ and $0.6 \mathrm{~V}$, respectively, ) while FMS required about 1.6 times the threshold stimulation voltage to reach its maximal response ( $140 \mathrm{~V}$ and $220 \mathrm{~V}$, respectively), indicating both modalities had a wide dynamic range. These 
observed graded responses are an important characteristic of any effective neural stimulation modality, as they directly relate to the capability of the modality to produce graded muscle contractions. Assuming the whole nerve is being excited with bipolar hook electrode stimulation, similar maximal levels indicate that most of the fibers in the nerve are being activated, which is important in generating useful forces. There is no evidence to suggest that FES and FMS have different intrinsic latencies, and any small differences in latency shown between the two can be attributed to a difference in stimulation location.

\section{B. Consistency and Selectivity of FMS}

The very low trial-to-trial variability of FMS further demonstrates its potential as an alternative to FES. Without this, there would be a much greater risk of stimulating at a higher or lower level than desired, producing unintended consequences.

Changing the position of the coil relative to the nerve had a clear effect on the evoked response as shown in Figs. 4 and 5. As the position of the coil was incremented from $4 \mathrm{~mm}$ to $6 \mathrm{~mm}$ to $8 \mathrm{~mm}$, the recruitment curve shifted to higher stimulation voltages by about $10 \mathrm{~V} / \mathrm{mm}$, but the nature of threshold, growth, and saturation of the recruitment curve did not change, as shown in Fig 4. This indicates that there were coil positions that allowed for fine tuning of selective muscle control. The relative selectivities of TA and LG could be modulated by changing coil position as displayed in Fig. 5. At $-16 \mathrm{~mm}$, TA was strongly activated at larger stimulation voltages while LG was not, while at $-12 \mathrm{~mm}$ LG was strongly activated at larger stimulation voltages while TA was not. It is worth noting that LG and TA are antagonist muscles, so activating them selectively is a critical first step in producing complex movements. In a clinical application it would not be possible to move the coil as we have done here, but multiple coils selectively activating particular muscles could be used instead.

\section{CONCLUSiOnS AND IMPACT}

We have shown magnetic stimulation of sciatic nerves of rodents and felines with small coils has some of the characteristics necessary for the viability of functional magnetic stimulation as an alternative to functional electric stimulation. FMS was able to produce graded responses, maximal levels of activation, and selective muscle activation when moved perpendicular to the length of the nerve, something the hook electrode could not do. Furthermore, FMS did not suffer from the primary drawback of FES: the need for a direct contact from the electrode to the tissue.

However, some significant drawbacks to FMS are also noted. FMS requires very large energies, on the order of $10 \mathrm{~J}$ to reach threshold levels of stimulation, compared to
$100 \mathrm{~nJ}$ for FES to reach threshold levels of stimulation. Two other drawbacks are a direct consequence of this: Long capacitor charging times, which reduces the maximum rate of stimulation, and unsafe heat levels, from the deposition of large energies without sufficient cooling time. Additionally, FMS requires relatively large coils that could not be feasibly implanted at the current size. All of these issues are obstacles for chronic implantable applications, but we believe that with smaller coils, the energies required to activate neural tissue will decrease due to a more focused excitation volume. This would greatly reduce the need for such high energy levels (i.e., allow lower voltages), and reduce the impact of capacitor charging time and thermal effects. Lower energy levels may allow methods of delivering stimulus energy without the need for a capacitor, substantially increasing the maximum stimulation frequency.

\section{REFERENCES}

[1] G. E. Loeb and R. A. Peck, "Cuff electrodes for chronic stimulation and recording of peripheral nerve activity.," J. Neurosci. Methods, vol. 64, pp. 95-103, Jan. 1996.

[2] A. Branner and R. A. Normann, "A multielectrode array for intrafascicular recording and stimulation in sciatic nerve of cats.," Brain Res. Bull., vol. 51, pp. 293-306, Mar. 2000.

[3] A. Barker, "An Introduction to the Basic Principles of Magnetic Nerve Stimulation," J. Clin. Neurophysiol., vol. 8, no. 1, pp. 26-37, 1991.

[4] X. Navarro, T. B. Krueger, N. Lago, S. Micera, T. Stieglitz, and P. Dario, "A critical review of interfaces with the peripheral nervous system for the control of neuroprostheses and hybrid bionic systems.," J. Peripher. Nerv. Syst., vol. 10, pp. 229-58, Sept. 2005.

[5] J. Wells, C. Kao, E. D. Jansen, P. Konrad, and A. MahadevanJansen, "Application of infrared light for in vivo neural stimulation.," J. Biomed. Opt., vol. 10, no. 6, p. 064003, 2010.

[6] X. Liu, D. B. McCreery, L. a. Bullara, and W. F. Agnew, "Evaluation of the stability of intracortical microelectrode arrays.," IEEE Trans. Neural Syst. Rehabil. Eng., vol. 14, pp. 91-100, Mar. 2006.

[7] J. M. Anderson, A. Rodriguez, and D. T. Chang, "Foreign body reaction to biomaterials.," Semin. Immunol., vol. 20, pp. 86-100, Apr. 2008.

[8] V. S. Polikov, P. A. Tresco, and W. M. Reichert, "Response of brain tissue to chronically implanted neural electrodes.," J. Neurosci. Methods, vol. 148, pp. 1-18, Oct. 2005.

[9] E. M. Wassermann, "Risk and safety of repetitive transcranial magnetic stimulation: report and suggested guidelines from the International Workshop on the Safety of Repetitive Transcranial Magnetic Stimulation, June 5-7, 1996.," Electroencephalogr. Clin. Neurophysiol. vol. 108, pp. 1-16, Jan. 1998.

[10] Z. Deng, S. H. Lisanby, and A. V. Peterchev, "Electric field depthfocality tradeoff in transcranial magnetic stimulation: simulation comparison of 50 coil designs," Brain Stimul., vol. 6, no. 1, pp. 1-13, 2013.

[11] G. Bonmassar, S. W. Lee, D. K. Freeman, M. Polasek, S. I. Fried, and J. T. Gale, "Microscopic magnetic stimulation of neural tissue.," Nat. Commun., vol. 3, p. 921, Jan. 2012.

[12] D. McRobbie, "Design and instrumentation of a magnetic nerve stimulator," J. Phys. E., vol. 18, no. 1, pp. 74-78, 1985.

[13] H. A. C. Wark, B. R. Dowden, P. C. Cartwright, and R. A. Normann, "Selective Activation of the Muscles of Micturition Using Intrafascicular Stimulation of the Pudendal Nerve," IEEE J. Emerg. Sel. Top. Circuits Syst., vol. 1, pp. 631-636, Dec. 2011

[14] F. Rattay, "Analysis of Models for External Stimulation of Axons," IEEE Trans. Biomed. Eng., vol. 33, no. 10, pp. 974-977, 1986. 